\title{
Relationship of Leptin Hormones with Body Mass Index and Waist Circumference in Saudi Female Population of the Makkah Community
}

\author{
Adil Omar Saeed Bahathiq*
}

Umm-Alqura University, Medical College, Departments of Physiology, Makkah, Saudi Arabia

\begin{abstract}
Objective: This study aims to study obesity in Saudi female population represented by Makkah community. Design: To study obesity, leptin concentration was measured and other measurements, like body mass index (BMI) and waist circumference (WC) have been used in this study. Setting: Departments of Physiology and Biochemistry, Medical College, Umm-Alqura Universty, Saudi Arabia. Subjects: Two-hundred and forty women $(n=240)$ between the ages 18 and 65 participate in this study. Volunteers were divided into three groups. The first group was the normal or control group with (BMI) range from 18 to 29.9, the second group was the obese and characterise with (BMI) $\geq 30$ and finally obese diabetic group with body mass index $(\mathrm{BMI}) \geq 30$ and suffer from diabetes mellitus. Main Outcome Measure: Leptin levels and Anthropometrics measurements. Results: Leptin was measured in all the groups and their means found to be $(8.4 \pm 1.4)$ in normal, $(56.3 \pm 18.8)$ in obese and $(42 \pm 19.3)$ in diabetic obese group. Leptin levels were directly associated with BMI in obese and diabetic obese group as follows: $(\mathrm{r}=0.350, \mathrm{p}=0.001)$, and $(\mathrm{r}=0.355, \mathrm{p}=0.001)$. Also, leptin concentrations were positively correlated with WC in obese and diabetic obese. Conclusion: Leptin concentrations were found to be high in both obese and diabetic obese group and showed a directly positive relation with BMI and waist circumference. Understanding that leptin hormone influencing appetite and body weight that cause obesity. However, understanding the relationship of leptin across all obesity categories may help in understanding pathophysiology and perhaps in developing the treatments for obese individuals.
\end{abstract}

Keywords: Obesity, female, leptin, diabetes mellitus, body mass index.

\section{INTRODUCTION}

Obesity is a condition of an abnormal or excessive accumulation of body fat in adipose tissue to the extent that health may be impaired [1]. It is a complex of multifactorial disease that develops from the interaction between genotype and the environment. However, it involves the integration of social, behavioural, cultural, physiological, metabolic, and genetic factors [2]. It is well established that directly or indirectly obesity is associated with various diseases, especially cardiovascular disease, hypertension, diabetes mellitus, sleep apnea, osteoarthritis, fatty liver disease, gallbladder disease, and certain types of cancer. Therefore, its manifestation poses a real threat to health [3].

Saudi Arabia, a Middle Eastern country with a population around 23 million, has undergone significant economic and cultural changes over the past thirty years. Approximately $60 \%$ of the population are urbanized and have adopted lifestyle reflecting their diet and physical activity. In Saudi Arabia the prevalence of obesity among female and, to a lesser extent, male adults has reached epidemic proportions. Obesity can be regarded as a major health problem among the Saudi population. A previous study indicated that in the overall population aged $14-70$ years, $13.05 \%$ of males and $20.26 \%$ of females were obese. This value is higher than that reported in the United Kingdom, Australian, Americans and Italian populations [4].

*Address correspondence to this author at the Umm-Alqura University, Medical College, Departments of Physiology, Alazeziah, P.O. Box: 6289; 3. Makkah, Saudi Arabia; Tel: 00966505579506; Fax: 009662-5364789; E-mail: adilobahathiq@hotmail.com
The hypothalamus plays a central role in the regulation of appetite, where the feeding center, located in the lateral hypothalamic nucleus, and the satiety center, located in the ventromedial nucleus, interact. The brain regulates energy homeostasis in response to signals from both adipose tissue and the gastrointestinal tract. The drive to eat and energy expenditure are adjusted so that over time, body weight remains stable [5]. Understanding the physiology of obesity, including the role of appetite as it relates to energy intake and weight gain, is essential for developing efficacious weight-loss therapies. There are also several compounds that appear to participate in the regulation of food intake, including circulating nutrients (e.g., glucose, amino acids, and fatty acids), metabolic compounds (e.g., lactate, pyruvate, and ketone bodies), and hormones (e.g., insulin, glucagon, cholecystokinin, leptin, and ghrelin) [6].

Leptin, (from the Greek word leptos, meaning thin) is a peptide hormone, secreted from adipose tissue, which influences energy homeostasis, immune and neuroendocrine function [7]. In humans, it is well established that plasma leptin levels are directly proportional to percentage body fat. Most obese individuals have high concentrations of leptin in their serum and plasma but exhibit leptin resistance because of decreased leptin transport into the central nervous system or downregulation of leptin receptors $[8,9]$.

Previous studies have evaluated leptin concentrations in small samples of lean and obese subjects [10]. However, preliminary data available on leptin concentration analysis across the range of BMI, did not use waist circumference (WC). Furthermore, the relationship between leptin level and 
WC have not been assessed in a sample of adults accounting for differences in age, fasting, physical activity and hypertension $[11,12]$.

The purpose of this study was to evaluate the relationship of leptin with BMI and waist circumference in a random sample of adult women classified as ranging from normal weight to severely obese based on BMI criteria. We anticipated a direct relationship of leptin with BMI and waist circumference.

\section{MATERIAL AND METHODS}

This study was submitted and approved by the University of Umm-Alqura Institutional Review Board, and all subjects signed an informed consent form.

Leptin hormone concentrations were analysed using Human leptin ELISA kit from Linco Research (6 Park Drive. St. Charles, Missouri 63304 USA). The analysis of Leptin was carried out by using DSX Automated ELISA System from (DYNEX Technologies .Inc.).

"BMI and WC" were measured as an anthropometric measurement.

\section{Subjects}

A total of 240 women aged 18-65 participated in this study. They were divided into three groups; (see below). After giving informed consent each participant completed a confidential. The study was carried out at Umm AL-Qura University, AL-Noor Specialist Hospital (Diabetic Centre) and King Abdul-Aziz Hospital (AL-Zaher). All blood analyses were carried out in the University Physiology Department and Professor Sultan research laboratory at Umm AL-Qura University.

First - control group (nonobese): 80 volunteers were recruited in this group, which had a BMI ranging from 18 to 29.99 .

Second - obese group: 80 volunteers were recruited in this group. Their BMIs were $\geq 30$.

First and Second Groups Met the Following Criteria:

1) Not on any diet or use any medication which can interfere with the result.

2) Not performing any physical activity, which may interfere with the result.

Third - diabetic obese group: 80 volunteers were recruited to this group. Their BMI were $\geq 30$.

\section{Anthropometric Measurements}

BMI was calculated as $\mathrm{kg} / \mathrm{m}^{2}$. Height was measured using a Harpenden anthropometer (Holtain, Ltd, Crymych, UK) to the nearest centimeter. Weight was measured using a Scale-tronix scale (Sharp Corp, Wheaton, IL,model 695, weighing to $364 \mathrm{~kg}$ ) to the nearest $0.1 \mathrm{~kg}$. Subjects were divided into five categories of BMI [2]. The categories were as follows: normal weight, $<25$; overweight, 25 to 29.9 ; obese I, 30 to 34.9 ; obese II, 35 to 39.9 ; severely obese, $>40$. No subjects had a BMI <18.5. Waist circumference was measured by locating the upper hipbone and placing a measuring tape around the abdomen (ensuring that the tape measure is horizontal). It should be comfortable and not cause compression of the skin [13].

\section{Hormone Levels}

Blood samples were collected after an overnight fast. Plasma were obtained by centrifugation of blood samples and stored at $-70^{\circ} \mathrm{C}$ at the Microbiology Department.

Following thawing of the samples, leptin levels were measured in duplicate using the human leptin Enzyme Linked Immunosorbent Assay kit. Leptin kits were purchased from the Millipore Corporation Research Inc (StCharles, MO, USA) and were used to test hormone levels at Professor Sultan research laboratory.

\section{Statistical Analysis}

Descriptive statistical analyses were performed using SPSS software (version 11.5, 2002). Pearson correlation coefficients were used to determine the relationship of BMI, waist circumference with leptin concentrations in these subjects groups (nonobese, obese and obese diabetic), while correlation was defined as a measure of the strength of a linear relationship between two variables. The statistical measure of linear association is known as the correlation coefficient, denoted by the symbol $\mathrm{r}$, and measures how close the points lie to a straight line. Its value always lies between -1 and +1 . The value +1 indicates a perfect positive relationship between the two variables and the value -1 indicates a perfect negative relationship. In addition, p-value is the probability of getting the observed difference, or one even more extreme, in the sample purely by chance from a population, where the true difference is zero. If the p-value is greater than 0.05 then, by convention, we conclude that the observed difference could have occurred by chance and there is no statistically significant evidence (at the 5\% level) for a difference between the groups in the population.

\section{RESULT}

The study samples were 240 women between 17 and 65 years old, with a mean age of $32.7 \pm 13.75$ years, height 1.54 $\pm 6.3 \mathrm{~m}$, and weight $74.9 \pm 17.5 \mathrm{~kg}$ (See Table 1).

\section{Leptin Concentration}

Mean of leptin concentrations in all three groups were found to be $8.4 \mathrm{ng} / \mathrm{ml}( \pm 1.4)$ in nonobese, $56.3( \pm 18.8)$ in obese and $42( \pm 19.3)$ in the diabetic obese group (See Table 1).

\section{BMI and Weight}

BMI was directly associated with weight and showed a strong positive relation $(r=0.935, P=0.000)$.

\section{BMI and Waist Circumference}

BMI was directly associated with waist circumference and demonstrated a strong positive relation $(r=0.840, P=$ 0.000) (See Fig. 1). 
Table 1. Summary of Characteristic Feature of all Volunteers in all Groups such as Age, BMI, WC, Weight, Height and Leptin Concentration

\begin{tabular}{|c|c|c|c|c|}
\hline \multicolumn{2}{|c|}{ Character } & Normal & Obese & $\begin{array}{c}\text { Obese } \\
\text { Diabetic }\end{array}$ \\
\hline Age & \multirow{3}{*}{ 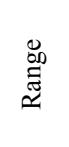 } & $18-38$ & $18-55$ & $20-58$ \\
\hline BMI & & $18-29.9$ & $30-53$ & $30-53.6$ \\
\hline WC & & $60-100$ & $77-149$ & $90-192$ \\
\hline Age (years) & \multirow{6}{*}{ 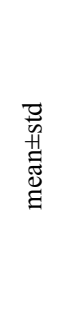 } & $20.1 \pm 2.3$ & $31.4 \pm 12.1$ & $46.6 \pm 7.9$ \\
\hline Height (m) & & $1.55 \pm 6.4$ & $1.556 \pm 6.4$ & $1.529 \pm 6.00$ \\
\hline Weight $(\mathrm{Kg})$ & & $55.61 \pm 7.9$ & $86.5 \pm 13.7$ & $82.7 \pm 10.2$ \\
\hline BMI & & $22.7 \pm 2.6$ & $35.7 \pm 5.2$ & $35.4 \pm 4.3$ \\
\hline WC (cm) & & $72.9 \pm 7.5$ & $101.6 \pm 12$ & $106.6 \pm 12.4$ \\
\hline \multirow{3}{*}{$\begin{array}{c}\text { Leptin } \\
\text { Concentration } \\
(\mathrm{ng} / \mathrm{mL})\end{array}$} & & $8.4 \pm 1.4$ & $56.3 \pm 18.8$ & $42 \pm 19.3$ \\
\hline & Max. & 10.99 & 99.60 & 97.27 \\
\hline & Min. & 5.12 & 8.21 & 12.71 \\
\hline
\end{tabular}

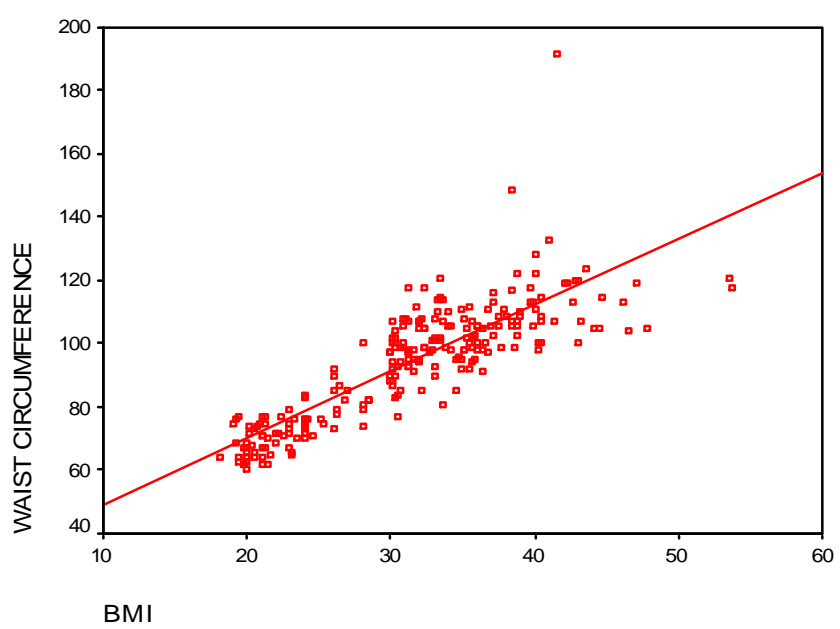

Fig. (1). Correlation between Waist Circumference and BMI.

\section{Leptin Concentration and BMI in Different Subject Groups}

\section{In Non-Obese Subjects}

The correlation between leptin concentration and body mass index was linear (data not shown).

\section{In Obese Subjects}

Leptin concentrations were directly associated with Body Mass Index and demonstrate a strong positive relation $(\mathrm{r}=$ $0.350, \mathrm{p}=0.001$ ) (See Fig. 2).

\section{In Diabetic Obese Subjects}

Leptin concentrations were directly associated with body mass index and showed strong positive correlation $(\mathrm{r}=$ $0.355, \mathrm{p}=0.001)($ See Fig. 3).

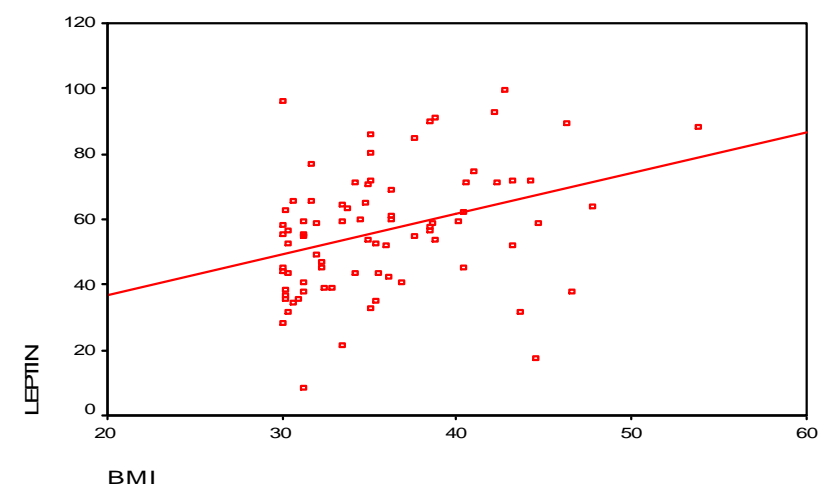

Fig. (2). Correlation between leptin concentration and BMI in obese subjects.

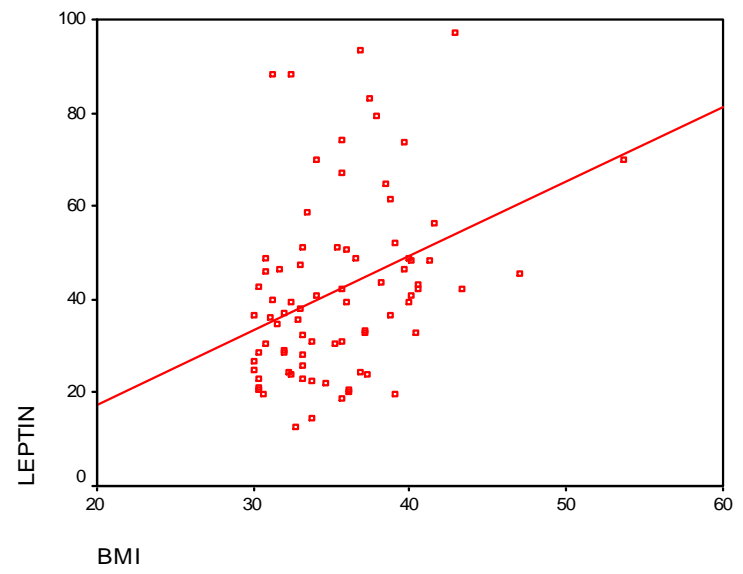

Fig. (3). Correlation between leptin concentration and BMI in obese diabetic subjects.

\section{Leptin Concentration and WC in Different Subject Groups}

\section{In Non-Obese Subjects}

There was no obvious correlation between leptin concentration and WC $(\mathrm{r}=0.115, \mathrm{p}=0.310)$ (data not shown).

\section{In Obese Subjects}

Leptin concentrations were directly associated with WC with a positive correlation $(r=0.299, p=0.007)$ (See Fig. 4).

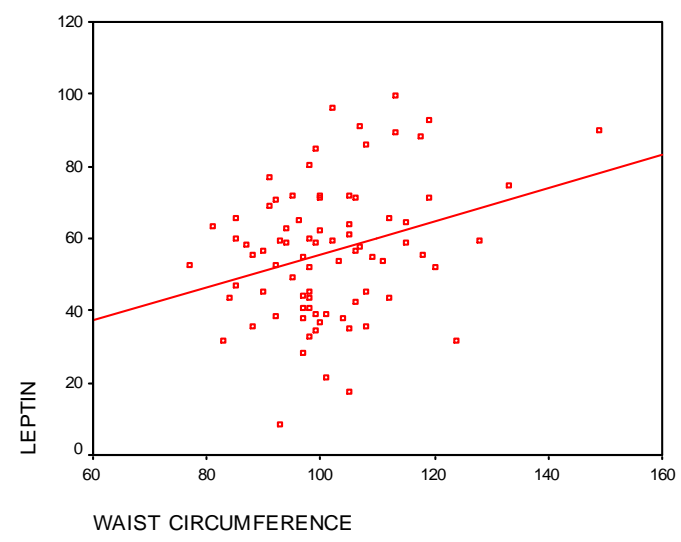

Fig. (4). Correlation between leptin concentrations with WC in obese subjects. 


\section{In Diabetic Obese Subjects}

Leptin concentrations were directly associated with $\mathrm{WC}$ and demonstrated a strong positive correlation $(\mathrm{r}=0.316, \mathrm{p}=$ 0.004) (See Fig. 5).

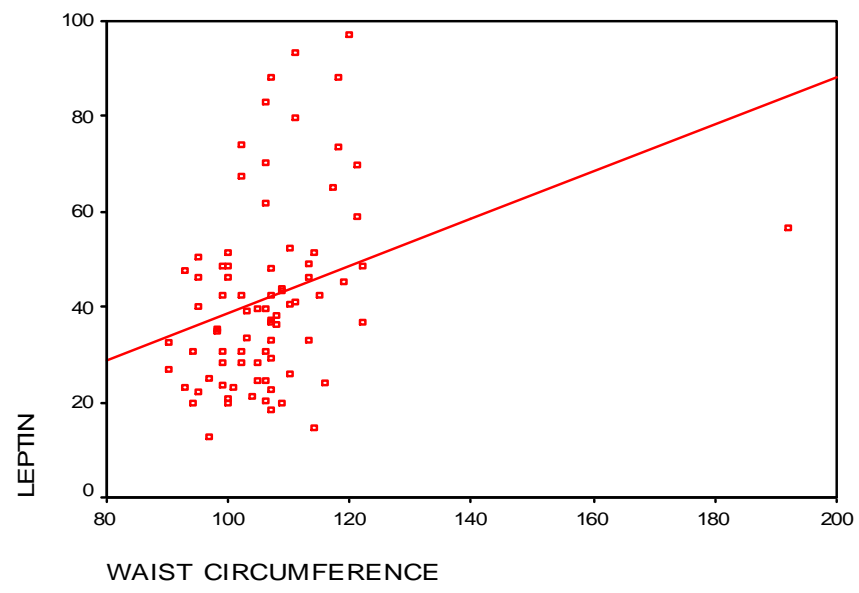

Fig. (5). Correlation between leptin concentrations and WC in obese diabetics.

\section{DISCUSSION}

The present work is the first to measure the relation between BMI, WC and leptin concentration to assess obesity in the Saudi female population. Leptin levels were directly associated with BMI and WC, and there were significant positive correlation between leptin and BMI categories.

Obesity is characterised by increased adipose tissue mass which results both from increased fat-cell number (hyperplasia) and increased fat-cell size [14]. Obesity is now extremely common in the world's population, and is replacing under-nutrition and infectious diseases as the most significant contributor to illhealth. Obesity currently affects $30 \%$ of the adult population in the United States and $35.5 \%$ in KSA [15].

The present study used the body mass index (BMI) and waist circumference (WC) as anthropometric measurement for obesity and fat content. Many studies have confirmed association between BMI and WC, with obesity and fat content. Body Mass Index correlates strongly with measurements of fat mass but it does not distinguish fat mass from lean mass [16]. In contrast, Waist Circumference provides a clinically acceptable measurement for assessing a patient's abdominal fat content [17].

\section{Obesity and Leptin Concentration}

This study was unique in that leptin concentrations were measured across the BMI rang in adult Saudi women taking into account several risk factors, including waist circumference, age diabetes and physical activity.

Previous studies found that there is little variation in leptin concentrations in the healthy communities according to nationality. A recent study indicated that leptin concen- trations in Omani control subjects was $10.6( \pm 4.2) \mathrm{ng} / \mathrm{ml}$ [18], and in American control subjects leptin concentrations was $7.8( \pm 0.7) \mathrm{ng} / \mathrm{ml}[19]$. In the present study, we found that the normal mean leptin concentration was $8.4( \pm 1.4)$ $\mathrm{ng} / \mathrm{ml}$ in Saudi women, which is equivalent to these values.

As previous studies have reported, leptin levels were higher in obese individuals than in normal-weight individuals [20, 21]. Regardless of weight, leptin seems to correlate better with subcutaneous fat rather than with visceral fat in both obese and non-obese subjects [22, 23]. The present study indicates that leptin concentrations were higher in obese subjects (See Table 1). This may be due to a diminished response in the leptin receptor signalling pathway, poor penetration of the blood-brain barrier by leptin, or due to the presence of a less active molecular forms of leptin [24]. In addition, the deficient leptin in nonobese subjects as compared to obese subjects is likely to be a target for leptin therapy, whereas obese subject with high leptin levels is likely to be resistant to leptin therapy [25]. Leptin resistance may occur directly as a result of obesity, but these may also be a lack of sensitivity to circulating leptin which could contribute to the aetiology of obesity [26].

The current study found that serum leptin concentrations were increased in relation to increased body fat content. The positive correlation between body fat and serum leptin is probably explained by the increased release of leptin from large fat cells. Furthermore, leptin can serve as an indicator of fat content and its level may be decreased by reduction of body fat even though BMI values remain unchanged [27].

Previous studies indicate that leptin is directly associated with insulin resistance [28, 29]. Insulin levels were not measured in this study. However, in the diabetic obese group, leptin concentrations were lower than in the obese group but still higher than the normal group, which is in accordance with other studies [30]. This may be due to differences in fat distribution between the obese diabetic and the obese non diabetic group. It has been shown that subcutaneous fat produces more leptin than visceral fat, and diabetics have more visceral fat and less subcutaneous fat. Another possibility is a relative insulin deficiency in the diabetic subjects, because insulin is an important stimulator of leptin production [30].

Uncontrolled diabetes is characterised by behavioural markers and metabolic concerns that arise as a consequence of profound insulin deficiency, including severe hyperglycaemia, depletion of body fat mass, and reduced circulating leptin levels that stimulate food intake [31]. It will be important to know whether the subcellular and tissue distribution of leptin is different in human adipocytes from lean and diabetic obese individuals and whether it can be altered by effectors, such as insulin [32].

There are high levels of leptin in diabetic obesity even although the level of glucose is controlled, because leptin antagonizes the action of insulin in the liver and the results are influenced by the changes in intrahepatic glucose with increased gluconeogenesis and decreased glycogenolysis. It is likely that these metabolic effects of leptin participate in the regulation of hepatic glucose metabolism under physiological conditions [33]. 


\section{OBESITY AND ANTHROPOMETRIC MEASURE- MENTS}

The body mass index (BMI) and waist circumference (WC) are simple and a less time-consuming test to perform in any clinical setting. BMI and $\mathrm{WC}$ were found to correlate well with leptin concentration in all subject groups in this study. This is due to the close relation between BMI, WC and body fat content in addition to the responsibility of visceral and subcutaneous fat for producing leptin $[34,35]$.

In our study, the mean BMI in nonobese, obese and diabetic obese groups were $22.7( \pm 2.8), 35.7( \pm 5.2)$ and $35.4( \pm 4.3)$, respectively. BMI increased with increasing weight and the data showed that BMI and leptin concentrations were higher in obese women than in other groups. The means of $\mathrm{WC}$ in the nonobese, obese and diabetic obese groups were $72.9( \pm 7.5), 101.6( \pm 12)$ and $106.6( \pm 12.4)$, respectively, showing that the high WC was found in the obese and diabetic obese more than in the normal group.

The present study found that there is a positive relationship between $\mathrm{BMI}$ and $\mathrm{WC}$, which means that any increase of BMI will be followed by increase in WC and leptin concentration (See Fig. 3).

\section{CONCLUSION}

This study showed a significant linear trend in total leptin, BMI and waist circumference. This study and others indicate that obesity and being overweight are enormous public health problems in Saudi Arabia especially for women.

The high prevalence of obesity among Saudi women could be attributed to limited physical activity as a result of the wide spread use of housemaids, the limited availability of exercise facilities for girls and women in Saudi Arabia and limited awareness in the population of the health risks associated with obesity.

\section{REFERENCES}

[1] "Obesity: preventing and managing the global epidemic. World Health Organ Tech Rep Ser 2000; 894.

[2] Al-Quaiz, Al-Joharah M. Current concepts in the management of obesity. An evidenced based review. Saudi Med J 2001; 22: 20510.

[3] Shigeta H, Shigeta M, Nakazawa A, Nakamura N, Yoshikawa T. Lifestyle, Obesity, and Insulin Resistance. Diabetes Care 2001; 24(3): 608 .

[4] El-Hazmi M, Warsy A. Prevalence of overweight and obesity in diabetic and non-diabetic Saudis. East Mediterr Health J 2000; 6: 276-82.

[5] Stanley S, Wynne K, McGowan B, Bloom S. Hormonal regulation of food intake. Physiol Rev 2005; 85: 1131-58.

[6] Havel PJ. Peripheral signals conveying metabolic information to the brain: Short-term and long-term regulation of food intake and energy homeostasis. Exp Biol Med 2001; 226: 963-77.

[7] Al-Harithy, Rowyda N. Relationship of leptin concentration to gender, body mass index and age in Saudi adults. Saudi Med J 2004; 25: 1086-90.

[8] Caro JF, Kolaczynski JW, Nyce MR, et al. Decreased cerebrospinal fluid/serum leptin ration in obesity: A possible mechanism for leptin resistance. Lancet 1996; 348: 159-61.

[9] Bjorbaek C, El Haschimi K, Frantz JD, Flier JS. The role of SOCS-3 in leptin signaling and leptin resistance. J Biol Chem 1999; 274: 30059-65.
[10] Hu FB, Chen C, Wang B, Stampfer MJ, Xu X. Leptin concentrations in relation to overall adiposity, fat distribution, and blood pressure in a rural Chinese population. Int J Obes Relat Metab Disord 2001; 1: 121-5.

[11] Tschop M, Weyer C, Tataranni AP, Devanarayan V, Ravussin E, Heiman ML. Circulating ghrelin levels are decreased in human obesity. Diabetes 2001; 50: 707-9.

[12] Rosicka M, Krsek M, Matoulek M, et al. Serum ghrelin levels in obese patients: The relationship to serum leptin levels and soluble leptin receptor levels. Physiol Res 2003; 52: 61-6.

[13] National Institutes of Health and National Heart Lung and Blood Institute Clinical guidelines on the identification, evaluation, and treatment of overweight and obesity in adults. The evidence report. Obes Res 1998; 6: 51S-209S.

[14] Camp H, S, Ren D, Leff T. Adipogenesis and fat-cell function in obesity and diabetes. Trends Mol Med 2002; 8: 442-7.

[15] Al-Nozha M, M. Al-Mazrou Y, Y. Al-Maatouq MA, et al. Obesity in Saudi Arabia. Saudi Med J 2005; 26: 824-89.

[16] Kopelman PG. Obesity as a medical problem. Nature 2000; 404: 635-43.

[17] Clinical guidelines on the identification, evaluation, and treatment of overweight and obesity in adults: executive summary. Expert Panel on the Identification, Evaluation, and Treatment of Overweight in Adults. Am J Clin Nutr 1998; 68: 899-917.

[18] Almaskari Y, Masoud Alnaqdy A. Adel. Correlation between serum leptin levels, body mass index and obesity in omanis. Sultan Qaboos Med J 2006; 6: 28-31.

[19] Veronica M, Carlson J. Joseph, Hunt C, Steven, Adams, D. Ted. Relationship of ghrelin and leptin hormones with body mass index and waist circumference in a random sample of adults. J Am Diet Assoc 2006; 106: 822-8.

[20] Considine RV, Sinha MK, Heiman ML, et al. Serum immunoreactive leptin concentrations in normal weight and obese humans. N Engl J Med 1996; 334: 292-5.

[21] Ruhl CE, Everhart JE. Leptin concentrations in the United States: Relations with demographic and anthropometric measures. Am J Clin Nutr 2001; 74: 295-301.

[22] Cnop M, Landchild MJ, Vidal J, et al. The concurrent accumulation of intra-abdominal and subcutaneous fat explains the association between insulin resistance and plasma leptin concentrations: Distinct metabolic effects of two fat compartments. Diabetes 2002; 51: 1005-15.

[23] Minocci A, Savia G, Lucantoni R, et al. Leptin plasma concentrations are dependent on body fat distribution in obese patients. Int J Obes Relat Metab Disord 2000; 24: 1139-44.

[24] Ostlund RE, Jr, Yang JW, Klein S, Gingerich R. Relation between plasma leptin concentration and body fat, gender, diet, age, and metabolic covariates. J Clin Endocrinol Metab 1996; 81: 3909-13.

[25] Adeyemi E, Abdulle A. A comparison of plasma leptin levels in obese and lean individuals in the United Arab Emirates. Nutr Res 2000; 20: 157-66.

[26] Wynne K, Stanley S, McGowan B, Bloom S. Appetite control. J Endocrin 2005; 184: 291-318.

[27] Masoud AY, Adel AA. Correlation between serum leptin levels, body mass index and obesity in Omanis. Sultan Qaboos Med J 2006; 6: 28-31.

[28] Donahue RP, Prineas RJ, Donahue RD, et al. Is fasting leptin associated with insulin resistance among non-diabetic individuals? The Miami Community Health Study. Diabetes Care 1999; 22: 1092-6.

[29] Liuzzi A, Savia G, Tagliaferri M, et al. Serum leptin concentration in moderate and severe obesity: Relationship with clinical, anthropometric and metabolic factors. Int $\mathrm{J}$ Obes Relat Metab Disord 1999; 23: 1066-73.

[30] Abdelgadir M, Elbagir M, Eltom M, Berne C, Ahren B. Reduced leptin concentrations in subjects with type 2 diabetes mellitus in Sudan. Metabolism 2002; 51: 304-6.

[31] Gelling WR, Joost O, Chistopher D. et al. Effect of uncontrolled diabetes on plasma ghrelin concentration and ghrelin-induced feeding. Endocrinology 2004; 145: 4575-82.

[32] Russell CD, Ricci MR, Brolin RE, Magill E, Fried SK. "Regulation of the leptin content of obese human adipose tissue." Am J Physiol Endocrin Metab 2001; 280: E399-404.

[33] Lisen L, Karkanias GB, Morales JC, et al. Intracerebroventricular leptin regulate hepatic but not peripheral glucose fluxes. J Biol Chem 1998; 273: 31160-7. 
[34] Maugeri D, Bonanno MR, Speciale S, et al. The leptin, a new hormone of adipose tissue: clinical findings and perspectives in geriatrics. Arch Gerontol Geriatr 2002; 34: 47-54.
[35] Ostlund RE Jr., Yang JW, Klein S, Gingerich R. Relation between plasma leptin concentration and body fat, gender, diet, age, and metabolic covariates. J Clin Endocrinol Metab 1996; 81: 3909-13.

Received: April 22, 2009

Revised: June 16, 2009

Accepted: June 16, 2009

(C) Adil Omar Saeed Bahathiq; Licensee Bentham Open.

This is an open access article licensed under the terms of the Creative Commons Attribution Non-Commercial License (http: //creativecommons.org/licenses/by$\mathrm{nc} / 3.0 /$ ), which permits unrestricted, non-commercial use, distribution and reproduction in any medium, provided the work is properly cited. 